\title{
A Literature Review of Individual Placement and Support (IPS) for Forensic Patients and Implications for Future Research
}

\section{Esther Dark ${ }^{*}$}

School of Allied Health and Social Care, University of Derby, UK

*Corresponding author: Esther Dark, Occupational Therapy Student, University of Derby, UK, E-mail: esthersamme@hotmail.co.uk

Received date: June 26, 2018; Accepted date: July 13, 2018; Published date: July 20, 2018

Copyright: @2018 Dark E, This is an open-access article distributed under the terms of the Creative Commons Attribution License, which permits unrestricted use, distribution, and reproduction in any medium, provided the original author and source are credited.

\begin{abstract}
The positive correlation between work and health is widely acknowledged and evidenced; and a strong predictor for successful community integration for forensic patients. Conversely, a lack of vocational opportunities progressively damages health, and potentially leads to disability, exasperation of mental illness and decreased life expectancy [1]. A review and literature search was undertaken to ascertain how vocational rehabilitation, specifically the individual placement and support model (IPS), within forensic settings can assist those transitioning from secure units to community living.
\end{abstract}

Methods: A literature search carried out between January-March 2018 comprised of literature a) published post-2008, b) in English, c) focused on forensic settings and/or forensic patients, and d) peer-reviewed literature from academic journals. Excluded literature included: unpublished results, such as protocols, supplements, dissertations, and poster presentations; primary research was prioritised over opinion pieces or secondary reviews. Following the Department for Work and Pensions (DWP) recommendations for improved employment support for those with mental health illness, legislation concerning greater work opportunities was successively introduced. Therefore, literature published post-2008 allowed for Government whitepapers based upon these

recommendations to be incorporated. In addition, it also reflects pertinent developments in the field of occupational therapy, following review, which criticised current forensic research as weak, lacking relevancy and methodological rigour.

Conclusion: IPS research has primarily utilised quantitative data, measuring job tenure and risk-reduction, rather than depth of experience of patients, which could greatly improve occupational therapy practice and service delivery. IPS' efficacy has been recommended within forensic institutions, yet little research exists for IPS' impact within community settings.

Keywords: Forensic patients; Individual placement and support; Vocational rehabilitation; Occupational therapy; Forensic populations

\section{Introduction}

The positive correlation between work and health is widely acknowledged and evidenced; and a strong predictor for successful community integration for forensic patients [1]. Conversely, a lack of vocational opportunities can result in occupational deprivation and imbalance [2-4]. Patients transitioning from secure care into the community face a multiplicity of challenges including: restrictive legalities, stigma associated to their mental health and offending history, loss of work-related skills, and low self-esteem [5], possibly inhibiting future employment. Consequently, patients with mental illness and offending histories are one of the most ostracised groups in society [6], and largely excluded from the UK workforce [7]. With greater work opportunities for those with mental illness high on the political agenda [8], and Government guidelines recommending services incorporate social inclusion principles [9], engagement in meaningful employment is high concern for community forensic services [10]. Nevertheless, despite the work aspirations of patients [11], research reveals typical employment activities in forensic settings lack purpose and challenge [5], inadequately preparing patients for the challenges of "real-work" and the capacity to manage associative risks.
As such, employment tenure upon discharge is low [12], leading to high rates of recidivism and readmissions.

Research within vocational rehabilitation has focused on the effectiveness of approaches [13], with supported employment, often synonymous with the internationally recognised Individual Placement and Support (IPS) model, proving the most successful in securing employment for those in mental health services [14]. Nevertheless, insufficient evidence exists for IPS' effectiveness within forensic practice [15]. Currently, several UK IPS trials are taking place to ascertain how IPS can be adapted to forensic settings; however, results are yet to be published. Based on the 'place-then-train' philosophy [16], IPS focuses on rapid job-placement, rather than lengthy pre-vocational preparation, described as "overprotective and paternalistic" [17]. IPS research has primarily utilised quantitative data, measuring job tenure and risk-reduction, rather than depth of experience, which could greatly improve service delivery for forensic populations.

\section{Literature Review}

Quantitative and qualitative study, evaluating the views of staff and patients in one high-secure-setting, Rampton Hospital [18], provided valuable insight into patients and professional's experiences concerning the importance of vocational activities. Despite recognition of their role in preventing reoffending, the study highlighted activities lacked 
“real-work"-essential for maintaining and developing patient's workrelated skill set. 276 patients out of a possible 324 were invited to participate, however, only $54.3 \%$ engaged, whilst $58 \%$ of the staff population participated. Mongione states, response-rates between $50 \%-59 \%$ are barely acceptable for self-administered postal questionnaires; given the questionnaires were administered face-toface, both response-rates are strikingly low, preventing robust generalisations. Furthermore, patient's self-reported questionnaires, identified by Bryman contain potential flaws: generalised questions, which can be meaningless for some respondents with little opportunity for elaboration and the potential for questions to be read incorrectly or in an incorrect manner. Due to the greater cognitive challenge presented by questionnaires, as opposed to face-to-face interviews, staff support was offered to mitigate patient's low literacy levels. Whilst enabling accurate completion, such support negated respondent anonymity, increasing social desirability bias; where patients may have felt pressured to respond positively in the presence of another. Identified activities to aid future employment were based upon a closed response question and patients were able "tick as many subjects as they wanted" from a given list; thus, minimising the opportunity to elaborate on meaningful activities. Given the weaknesses incurred through the methodology, research utilising an alternative approach, such as inductive analysis, rather than the study's deductive thematic analysis, could further explore beneficial features of vocational support to improve practice. When making generalisations relating to the quality of vocational programmes in forensic settings, although the study presented a balanced view of both staff and patients, it only reflected those from high-secure-settings, where access to employment is notoriously low due to high-levels of risk and security measures imposed. IPS, therefore, may not be achievable for this population where patients are typically moved to low-secure settings, with little immediate employment [19].

Beck and Wernham's [5] evaluation of employment opportunities across one medium-secure unit, similar to the findings from Vollm et al. [18], discovered activities offered no "scope for progress", arguing skill development is crucial for future employment. Subsequently, several enterprises, including cafes and shops, underpinned by the IPS framework, were established. The only criterion for referral: a motivation to work. However, as evidenced by Vollm et al. [18], discrepancies can exist between patient's perceptions of ability, whereby patients possibly overestimate their level of skill. Of the 137 who participated in work-tasters, only 33 secured employment, this inconsistency was un-explored. Nevertheless, qualitative feedback, based upon patient's service-evaluations, customer feedback and clinical reports revealed an increase in patient's self-efficacy, and sense of empowerment; thus "counteracting the stigma of the passive patient" often prevalent in such settings. However, as jobs were unpaid and delivered on-site, with "a careful balance... between security and risk taking" and staff to patient ratios strictly observed, the authenticity of a real-work context was limited. Nonetheless, the opportunity to engage in work-experience, cultivated valuable skills, supporting the possibility of securing employment upon discharge.

Craik et al. [20] ethnographic study of twenty-six patients explored the occupational engagement in medium and low-secure settings through five focus groups. Despite barriers to participation tempered by risk implications, environmental constraints and staff shortages, the study highlighted patient's desire for relevant work preparation, which could provide them with the motivation to move forward. Therefore, it appears counter-productive to constrain vocational rehabilitation to lengthy prevocational training, which dismisses patient's work aspirations and hopes for the future. Occupational therapists were identified as key for enabling relevant activities. Indeed, IPS' recognised benefits of client-centeredness and acknowledgement of productive occupations, echo the central tenets of occupational therapy [21]; by augmenting therapeutic practice alongside employment-support, which champions recovery principles, occupational therapists can be significant in embedding IPS [22]. Yet despite the priority of supported-employment within the realm of occupational therapy, IPS remains under-practiced, distancing itself from traditional more familiar practices.

Samele et al. [15] service-evaluation of an employment and social inclusion pilot evidenced how vocational opportunities for those in less-secure settings can be attained successfully, with flexible one-toone support identified as essential for supporting patient's return to employment; a feature of IPS. The project, a paid community-based painting and decorating programme, initially underpinned by IPS principles; yet due to practical difficulties, not identified in the publication was "scrapped", resulting in an alternative approach. Quantitative (number securing employment) and qualitative (staff and patient interviews) data was collected over a three-year period. Of the 87 referrals, 57 patients were involved (65\%); enough to make generalisations. Most participants were white British males (93\%); although a high proportion of males, the sample is assumedly representative as secure populations tend to be inhabited by younger males (88\%). However, research with a broader demographic including the needs of women, identified by Baker and McKay, would enable greater understanding of what constitutes 'mental health' and 'work' across cultures and genders [13]. It is important to note positive qualitative findings maybe over-exemplified due to the purposive sample recruited for patient interviews $(\mathrm{n}=18)$, which were based upon regular attendance and engagement with the project, thus excluding patients who did not find the project beneficial. Furthermore, due to the exploratory nature, the evaluation omitted rigorous assessments, which McQueen [23] suggests is crucial for measuring outcomes and improving care.

Further evidence concerning less-secure settings is obtained from Roberts et al. study [24], exploring two community-based schemes: one promoting vocational skills; the other, healthier living. The vocational project offered educative outdoor activities through a notfor-profit organisation, where accredited qualifications were awarded on completion. The project adopted an inclusive approach, whereby all patients with vocational aspirations could participate-thus findings are assumedly representative of the whole forensic unit. Of the 44 patients, 35 had not maintained employment over the last decade, however this could be explained by lengthy detentions. Findings attained from staff reports, patient feedback, attendance, and observations, indicated developing community work-based activities in low-secure settings can be achieved through planned positive risk taking. Indeed, IPS can produce effective work patterns, as well as adaptive coping strategies [25]. Yet such success was predominately based upon attendance, rather than patient's direct qualitative feedback and motivations for attending. It is important to note, as evidenced in Farnsworth et al. [26] study, patients often attended therapy groups to appease staff, or "kill ... time" and Vollm et al. [18] study, where reasons included "getting off the ward" and "feeling obliged". Nevertheless, following project completion, the authors stated many "discussed plans to seek vocational opportunities". Researcher bias may exist as the evaluation was based chiefly upon the authors' observations, who were also the patient's occupational therapists. Therefore, supporting staff may have felt no choice, but to ethically intervene during activities, reducing 
integrity of findings. Furthermore, due to the nature of the evaluation, which primarily concerned itself with the project's feasibility and sustainability, it excluded depth of patient experience. Therefore, the authors concur, future research should focus on qualitative experiences of such programmes. As no participants engaged in "aggressive or violent behaviour", the authors concluded participation in positive risk-taking through employment was effective. However, this conclusion highlights the limitations of observations which focus on behaviours, and not intentions. Understanding and measuring risk is much more than physical behaviour, rather as [27] cross-sectional survey, concerning occupational therapist's understanding of risk, identifies risk as: "sharing responsibility ... and increasing autonomy". Due to the supported individualised risk assessments, determining how patients would sustain engagement in further training or manage related risks unaccompanied in community-settings requires exploration.

Smith et al. [28] evaluation concerning a pilot work-project for a forensic learning disability practice, highlighted the impact of work for supporting community transitions. Unlike lengthy in-house vocational rehabilitation, the programme provided graded progression into the community. Based upon un-structured interviews with four participants, staff observations, attendance patterns and journal reports; findings revealed, patients increased motivation, self-esteem and confidence. However, due to the small-scale reflective nature of the study and lack of structured assessments, robustness of findings, and project replication is challenging; as the authors concur, quantitative data could support findings. Nevertheless, the use of participant's journals was beneficial, not only providing a "rich source of data", offering insight into "past experiences... identity... aspirations and ... personality", but also enabled participants to reflect on their achievements during the project.

McQueen and Turner's [29] exploration of forensic patient's views of work, found work provided "gradual steps" towards community life, thus further evidencing the significant role work plays in successful community transitions. Through a phenomenological lens, involving semi-structured interviews, ten purposively selected participants from a range of forensic settings, found work promoted recovery by "normalising life" and "facing barriers". A purposive sample, may however give rise to a highly motivated cohort; thus, favouring positive findings. Additionally, due to the phenomenological approach, findings were also open to the researcher's own subjective interpretation, affecting both process and analysis. Yet, phenomenological research is not intended to provide generalisations, instead it aims to provide valuable insight into the lived experience.

Tregoweth et al. [30] hermeneutic study, exploring eight patient's experiences of re-entering the workforce following discharge, through un-structured interviews, described work as "transformative": A place to practice skills, resulting in personal accomplishment and development of self-management strategies thus decreasing risk. However, due to issues of disclosure, reconciling past incarcerations, increased feelings of "being set apart", indicating the possibility of future problems concerning stigma and isolation. Further research is needed into how such obstacles are surmountable upon discharge, and prevent exacerbation of psychiatric symptoms, a criticism of IPS cited by Burton et al. [31]. The study set in New Zealand, demonstrates the benefits of IPS overseas; however, differences in employment climates and UK forensic-settings may impact generalisations to UK settings. Indeed, Garabette and Burns [17] consider IPS' value in Europe less certain, identifying Europe's less work-orientated culture, impenetrable labour market, and better welfare benefits, therefore less financial incentive to participate, as barriers. This suggests a similar UK-based study, examining the experience of patient's return to work journey, would be of importance.

Royal College of Occupational Therapists [32] encourages for future research to concern itself with how forensic patients manage transitional periods, "so patients feel better equipped to cope and not panic!". However, scant research exists focusing on patient's transitions from deviant status' to developing new social identities, which can be formed through employment [5,33]. Coffey [33] notes, such identity formation is essential for smoothing transitions. Much of the literature explored supported employment within secure settings, as opposed to its efficacy in community settings, where the opportunity to engage in deviant occupations, or not participate in compulsory programmes is of higher risk [34]. Samele et al. [15] argues a vast chasm exists between teaching employment skills, and job retention, furthermore, discrepancies in community employment support is patchy.

Thus, future research should seek to add to the the body of IPS' existing research base, by exploring the effectiveness of IPS in supporting patients transition from forensic institutions into the community-considered to be the riskiest period for forensic populations [35]. Research should look beyond measurable outcomes of job attainment, and aim to understand the wider implications of patient's experiences of IPS regarding community integration and selfmanagement strategies. Blank et al. [13] review discovered the voice of patients are wholly underrepresented in forensic research; yet such qualitative research is crucial in enhancing service delivery [36-39].

\section{Implications for Occupational Therapy Practice}

As a body of professionals, committed to a client-centred approach, future occupational therapy research should aim to understand what works well with this vulnerable cohort returning to community life. The valuable insight derived from patient experience can further understand barriers and supporting aspects of IPS, thus enabling professionals to improve care pathways from high-secure institutions to effective work-placements in the community.

\section{References}

1. Waddell L and Burton A (2006). Is work good for your health and wellbeing? London: HM Stationary Office.

2. HM Government (2009) Work, Recovery and Inclusion: Employment support for people in contact with secondary care mental health services.

3. O'Connell M and Farnworth L (2007) Occupational therapy in forensic psychiatry: A review of the literature and a call for a united and international response. Br J Psychiatry 70: 184-191.

4. Farnworth L, Munroz, JP (2001) An occupational and rehabilitation perspective for institutional practice. Psychiatr Rehabil J 32: 192-8.

5. Beck C, Wernham C (2014) Improving access to competitive employment for service users in forensic psychiatric units. BMJ Qual Improv Rep 3: u204182.

6. Coffey, M (2006) Researching service user views in forensic mental health: A literature review. J Forens Psychiatry Psychol 17: 73-107.

7. Department of Health (2006) Vocational services for people with severe mental health problems: Commissioning guidance. London.

8. https://www.england.nhs.uk/wp-content/uploads/2014/10/5yfv-web.pdf Accessed on 17.02.2018

9. Harrison D, Sellers A (2008) Occupation for mental health and social inclusion. Br J Occup Ther 7: 216-18. 
10. Couldrick L, Alred D (2003) Forensic Occupational Therapy, Philadelphia: Whurr Publishers Ltd.

11. Centre for Mental Health (2013) Briefing 47: Barriers to employment.

12. Davies S, Clarke M, Hollin C, Duggan C (2007) Long-term outcomes after discharge from medium secure care: a cause for concern. Br J Psychiatry 191: 70-74.

13. Blank A, Harries P, Reynolds F (2011) Mental health service users perspectives of work: A review of the literature. Br J Occup Ther 74: 191-199.

14. Kinoshita Y, Furukawa TA, Kinoshita K, Honyashiki M, Omori IM, et al. (2013) Supported employment for adults with severe mental illness. Cochrane Database Syst Rev CD008297.

15. Samele C, Keil J, Thomas S (2009) Securing employment for offenders with mental health problems, Towards a better way, policy paper, Sainsbury Centre for Mental Health.

16. Sainsbury Centre for Mental Health (2009) Briefing 37: Doing what works Individual placement and support into employment.

17. Garabette J, Burns T (2009) Individual Placement and Support: Helping People with Severe Mental Illness Get Real Jobs. International Handbook of Occupational Therapy Interventions, NY: Springer Science and Business Media.

18. Völlm B, Panesar P, Carley K (2014) Promoting work-related activities in a high secure setting: exploration of staff and patients' views. J. Forens. Psychiatry Psychol 25: 26-43.

19. Lockett H, Secker J, Grove B (2005) Participants' and their clinicians' perceptions of the impact of having access to work opportunities through the first step trust (FST) project in Broadmoor (Research report). London: Sainsbury Centre for Mental Health.

20. Craik C, Bryant W, Ryan A, Barclay S, Brooke S, et al. (2010) A qualitative study of service user experiences of occupation in forensic mental health Aust Occup Ther J 57: 339-344.

21. Baxter C, Prior S, Forsyth K, Maciver, D, Meiklejohn A, et al. (2012). Mental health vocational rehabilitation - occupational therapists perceptions of individual placement and support. Int J Ther Rehabil 19: 217-226.

22. Priest B, Bones K (2012) Occupational therapy and supported employment: is there any added value? Ment Heal Soc Inc 16: 194-200.

23. Mcqueen, J (2011) Towards Work in Forensic Mental Health, National Guidance for Allied Health Professionals, NHS Scotland.

24. Roberts C, Davies J, Maggs G, R (2015) Structured community activity for forensic mental health - a feasibility study. J. Forensic Prac 17: 180-191.

25. Prior S, Maciver D, Forsyth K, Walsh M, Meiklejohn A et al. (2013) Readiness for Employment: Perceptions of Mental Health Service Users. Community Ment Health J 49: 658-667.
26. Farnworth L, Nikitin L, Fossey E (2004) Being in a secure forensic psychiatric unit: Every day is the same, killing time or making the most of it. Br J Occup her 67: 430-438.

27. Connell C (2016) Forensic occupational therapy to reduce risk of reoffending: a survey of practice in the United Kingdom, J Forens Psychiatry Psychol 6: 907-928.

28. Smith A, Petty M, Oughton I, Alexander R (2010) Establishing a WorkBased Learning Programme: Vocational Rehabilitation in a Forensic Learning Disability Setting. Br J Occup her 73: 431-436.

29. Mcqueen J, Turner J (2012) Exploring forensic mental health service user's view on work: an interpretive phenomenological analysis. J. Forensic Prac 14: 168-179.

30. Tregoweth J, Walton J, Kirk R (2012) he experiences of people who re-enter the workforce following discharge from a forensic hospital. J Vocat Rehabil 37: 49-62.

31. Burton ZC, Vella C, Twamley WE (2009) Supported Employment for Individuals with Severe Mental Illness in I, Soderback International Handbook of Occupational herapy Interventions NY: Springer Science and Business Media.

32. Royal College of Occupational herapists (2017) Occupational herapists' Use of Occupation Focused Practice in Secure Hospitals, Practice guideline, Second edition.

33. Coffey M (2012) A risk worth taking? Value differences and alternative risk construction in accounts given by patients and their community workers following conditional discharge from forensic mental health services. Health Risk Soc 14: 5.

34. Centre for Mental Health (2011) Pathways to unlocking secure mental health care. National Mental health Development Unit

35. Joint Commissioning Panel for Mental Health (2013) Guidance for commissioners of forensic mental health services Practical mental health commissioning.

36. Drennan G, Wooldirdge J, Aiyegbusi A, Alred D, Ayres J, et al. (2014) Making recovery a reality in forensic settings, Centre for Mental Health, Mental Health Network NHS Confederation.

37. https://www.centreformentalhealth.org.uk/Handlers/Download.ashx? IDMF=e94d8999-4010-4a5e-a5d8-0c3f1eb2d0e6

38. https://www.gov.uk/government/uploads/system/uploads/ attachment_data/file/228818/7742.pdf

39. Dunn C, Seymour A (2008) Forensic psychiatry and vocational rehabilitation: where are we at? Br J Occup her 71: 448-450. 